\title{
Antimicrobial Usage and -Resistance in Livestock: Where Should We Focus?
}

\author{
loannis Magouras ${ }^{1 *}$, Luís P. Carmo ${ }^{1}$, Katharina D. C. Stärk ${ }^{2}$ \\ and Gertraud Schüpbach-Regula ${ }^{1}$ \\ ${ }^{1}$ Veterinary Public Health Institute, Vetsuisse, University of Bern, Bern, Switzerland, ${ }^{2}$ SAFOSO AG, Bern, Switzerland
}

Keywords: antimicrobials, resistance, livestock, public health, One-Health

Antimicrobials represent one of humanity's medical revolutions enabling us to treat both human and veterinary bacterial infections. It is, therefore, of utmost importance to preserve their effectiveness. However, during the last decades, the continuing rapid development of antimicrobial resistance (AMR) has emerged as a major global public health concern (1). Resistant bacteria may hamper the treatment of infections resulting in prolonged illness, disability, and death (2).

In veterinary medicine, antimicrobials play a crucial role in the maintenance of animal health, animal welfare, and food-safety (3). However, a not yet quantifiable share of the burden of resistance for public health is attributable to the use of antimicrobials in livestock production (4-6). Farm animals are exposed to considerable quantities of antimicrobials (7) and can act as an important reservoir of AMR genes, which could be transmitted to humans through the food chain, direct animal contact and the environment. Use of antimicrobials in agriculture also includes those defined by the World Health Organization (WHO) as "critically important" for human medicine (8). Resistance against these substances can limit dramatically the treatment options against serious human bacterial diseases. Notorious examples include the vancomycin-resistant enterococci (VRE), the extended-spectrum $\beta$-lactamase (ESBL) producing Enterobacteriaceae and the recently detected plasmid-mediated colistin resistance (mcr-1 gene) in livestock, food, and humans in China (9-11).

Resistant bacteria can be introduced into the environment through several ways, such as the land application of livestock manure as fertilizer (12). The globally rising aquaculture sector, which is characterized by extensive use of antimicrobials, represents another important source of resistant bacteria that can find their way into the environment (13). Our understanding on the epidemiology of AMR in livestock production is also hampered by the lack of comprehensive antimicrobial usage (AMU) data in the majority of countries. Furthermore, AMR development and spread is driven by human behavior, from the prescription of antimicrobials to infection prevention and control. Understanding these factors is a major step toward fighting against AMR.

The complex epidemiology of AMR emphasizes the need for highly interdisciplinary research approaches, comprising humans, animals, and the wider environment. In line with the WHO global action plan on AMR (14), it is the authors' opinion that research should be prioritized toward (a) understanding the social/behavioral drivers of AMU and AMR, (b) establishing or improving systems to monitor AMU, and (c) encouraging a holistic approach through the One-Health concept when addressing the phenomenon and risk of AMR.

Published: 15 September 2017

Citation:

Magouras I, Carmo LP, Stärk KDC and Schüpbach-Regula G (2017) Antimicrobial Usage and-Resistance in Livestock: Where Should

We Focus?

Front. Vet. Sci. 4:148.

doi: 10.3389/fvets.2017.00148

\section{SOCIAL SCIENCES}

It is well established that resistance to a new antimicrobial substance begins shortly after its introduction; therefore, development of new antimicrobials should not be viewed as the only solution to combat AMR (15). The emergence and spread of AMR is largely influenced by human behavior, which in turn is shaped by cultural, social, political, and economic factors (16). This is also evident 
in the wide variation across the globe in patterns of use and resistance to antimicrobials, which cannot always be explained by differences in the diseases present, in health care infrastructure or farming systems $(17,18)$. Therefore, social sciences can shed light on the multi-faceted reasons that lead to the application of antimicrobials and the development of AMR. Social sciences are also valuable in identifying the most impactful and feasible interventions to counteract the AMR phenomenon.

In livestock production, veterinarians and farmers play a preponderant role when it comes to AMU and AMR. In many cases, veterinarians decide whether to treat an animal or not with antimicrobials, select the antimicrobial to be used, as well as define the dosage and route of administration. Veterinarians also advise farmers on animal health, biosecurity and production management issues that can strongly influence animal health, AMU, and the transmission of resistant bacteria. Farmers are a source of valuable information on farm management, biosecurity, animal health, and welfare that could be used to identify risk factors (and consequently interventions) associated with AMU in livestock.

Surveys and expert opinions are well-accepted approaches for exploring the behavioral basis of AMU and AMR. These methods could provide informative data on the attitudes, motivation, and knowledge of veterinarians and farmers toward AMU and AMR (19). On the other hand, controlled experimental studies that assess the success of specific interventions are rarely conducted. This research area should be expanded to lay the foundations for the design and implementation of intervention strategies toward the reduction of AMU and AMR.

\section{MONITORING OF AMU}

Bacteria can be naturally resistant against specific antimicrobial classes (intrinsic resistance) (20), however in the majority of the times, it is the exposure to antimicrobials that provide the necessary selective pressure for the emergence and spread of resistant bacteria. It should be emphasized that non-antimicrobial agents, namely metals and biocides are also implicated in co-selection of AMR (21). Data collection on AMU is an indispensable step in our attempt to understand and fight AMR. Monitoring of AMU allows the analysis of temporal trends in antimicrobial consumption and can ensure compliance with prudent usage practices, programs, or regulations. Furthermore, monitoring systems can assist in identifying the most efficient interventions for optimizing AMU. In combination with AMR data, quantification of AMU can be useful not only in detecting risk factors for the emergence of resistance, but also in describing temporal associations between AMU and AMR. This would provide evidence on the link between AMU and AMR to researchers, as well as policy and decision makers. In addition, analyzing these data can provide a basis for targeted research and development. The need for standardized usage data of high quality and resolution has been stressed by the European Surveillance of Veterinary Antimicrobial Consumption (ESVAC) $(22,23)$. The abovementioned benefits of monitoring antimicrobial consumption can be boosted when data on consumption per species are available. However, the resource-demanding nature of such monitoring systems often combined with political and confidentiality issues explains why only a few countries, such as The Netherlands and Denmark, have nation-wide automated monitoring systems in place $(24,25)$. Monitoring systems that are based on the collection of farm level data allow for the implementation of benchmarking strategies. These make it possible to rank individuals (farmers or veterinarians) by their level of AMU and to implement measures in order to reduce consumption by the top users. This is an additional benefit, given that benchmarking strategies have been quite successful in reducing AMU in the countries that adopted them. Denmark and The Netherlands are among the countries which experienced a drop in antimicrobial consumption following the implementation of benchmarking systems $(26,27)$. Alternatives to automated systems include performing randomized field studies or extrapolating species' consumption through sales data stratification (28). Nonetheless, automated collection of prescription/usage data should be preferred as long term goals.

\section{THE ECOLOGY OF AMR AND THE NEED FOR A ONE-HEALTH APPROACH}

The complex epidemiology of AMR together with the socioeconomical drivers make this topic the quintessential OneHealth issue. Transectoral and transdisciplinary approaches are a "must-do" to tackle AMR appropriately. A reduction in AMU was not always followed by a decline in AMR, as demonstrated in the case of VRE (6). Reducing the dissemination and transmission of resistant bacteria within and between animal and human populations is central when aiming to fight AMR. The ability of bacteria to disseminate from one setting to another, sometimes over large geographic distances and among the different populations, makes it difficult to explain with certainty the origin of resistant bacteria strains. Therefore, the reservoirs and the transmission pathways of antimicrobial-resistant bacteria merit further investigation, ideally through a One-Health approach.

Livestock trade creates a complex, heterogeneous, contact network that shapes between-herd transmission of infectious diseases. Direct transmission of resistant bacteria is well documented for livestock-associated methicillin-resistant Staphylococcus aureus (LA-MRSA). Here, animal trade has been identified to be a major driver of LA-MRSA dissemination $(29,30)$. For other bacteria, such as Enterobacteriaceae and in particular Escherichia (E.) coli, fecal shedding represents the main route of dissemination, thus not only host, but also environmental reservoirs may exist which constitute multiple, complex ways of resistance introduction and transmission. So far, experimental studies have demonstrated animal-to-animal transmission of resistant E. coli under controlled conditions within confined compartments (31). However, potential factors that drive transmission, such as farm management and the farm environment, have not been studied thoroughly for bacteria such as E. coli or Enterococci. The practice of land application of livestock slurry and manure represents a major source for introduction of resistant bacteria into the environment $(12,21)$. Animals can as well excrete resistant bacteria 
directly in the environment through their feces while being on pastures (32). E. coli spends approximately half of its life cycle in the external environment and, therefore, anything contaminated with these potentially antimicrobial-resistant bacteria may constitute a reservoir for their dissemination (33).

Wild animals are usually not treated with antimicrobials; however, they can carry antimicrobial-resistant bacteria from the farm's surrounding contaminated environments. Wild animal species that acquire resistant bacteria could constitute an additional reservoir of AMR in the environment and could function as vectors (and eventually as amplifiers) for dissemination to other species, including humans (34).

It is, therefore, important to improve our knowledge on how animal contacts and trade (direct transmission), farm management, and the wider farm environment (indirect transmission) drive the dissemination of AMR and to identify potential interventions to counteract this phenomenon. Farm management studies could include all those practices that potentially facilitate spread of resistant bacteria within and between farms and from farms to the environment, such as farm hygiene and biosecurity, animal waste management, structure (and construction material) of holdings as well as animal production intensity.

Holistic, One-Health approaches should always be backed with molecular epidemiological data, which can provide information about links between resistance genes observed in different samples, such as from animals of different origin. Resistance genes should be studied not only in animal samples but also in the wider farm environment, such as farmers, other livestock species, farm pets, wildlife, manure, and water. These ecological data can provide the molecular link to characterize reservoirs of resistant bacteria and could support studies on transmission pathways between animal populations but also from animals to humans and vice versa. Source attribution can be of help to shed light on the contribution of AMR originating from livestock to the public health resistance burden. Moreover, it can also be an important piece of evidence when developing targeted interventions against AMR. Genomic data might also provide some additional information on potential evolutionary processes in bacteria during transmission within the studied populations. Furthermore, molecular epidemiology data can shed some light on how much of the resistance reservoir is attributed to the spread of resistant bacteria or de novo emergence due to AMU selection pressure in the studied farms.

\section{REFERENCES}

1. WHO. Antimicrobial resistance. Bull World Health Organ (2014) 61:383-94. doi:10.1007/s13312-014-0374-3

2. de Kraker MEA, Davey PG, Grundmann H. Mortality and hospital stay associated with resistant Staphylococcus aureus and Escherichia coli bacteremia: estimating the burden of antibiotic resistance in Europe. PLoS Med (2011) 8:e1001104. doi:10.1371/journal.pmed.1001104

3. FAO. The FAO Action Plan on Antimicrobial Resistance. Rome: Food and Agriculture Organization of the United Nations (2016). p. 3-25. Available from: http://www.fao.org/3/a-i5996e.pdf

4. Chang Q, Wang W, Regev-Yochay G, Lipsitch M, Hanage WP. Antibiotics in agriculture and the risk to human health: how worried should we be? Evol Appl (2015) 8:240-7. doi:10.1111/eva.12185

\section{CONCLUSION AND PROSPECTS}

AMR is a complex phenomenon and is driven by biological processes and socio-economical factors. Understanding the attitude and knowledge of farmers and veterinarians toward AMU and AMR is a crucial step for the design of strategies to combat this public health threat. The lack of detailed AMU data impacts our ability to interpret surveillance data on AMR and to design efficient interventions. Therefore, monitoring systems to fill this knowledge gap should be prioritized. Finally, the ecology of AMR should be addressed with a holistic, One-Health approach combining expertise from different disciplines, such as veterinary clinicians, public health scientists, microbiologists, wildlife veterinarians, environmental scientists (ecologists), agricultural/forestry scientists, and epidemiologists.

\section{AUTHOR CONTRIBUTIONS}

IM wrote the manuscript. LPC provided valuable expertise on monitoring systems for antimicrobial usage and social sciences. KS provided valuable expertise on the topics of One-Health and social sciences. GS-R provided valuable expertise and feedback in all topics included in this opinion manuscript and assisted IM in the conceptualization of the manuscript. All the authors have read and approved the manuscript.

\section{ACKNOWLEDGMENTS}

This manuscript summarizes IM's contribution during the workshop on Digital Transformation of Animal Health Data, which was sponsored by the OECD Co-operative Research Programme: Biological Resource Management for Sustainable Agricultural Systems whose financial support made it possible for the author to participate in the workshop.

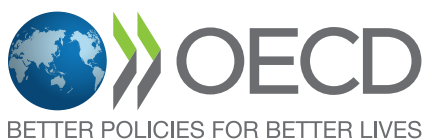

Disclaimer: The opinions expressed and arguments employed in this publication are the sole responsibility of the authors and do not necessarily reflect those of the OECD or of the governments of its Member countries.

5. Garcia-Alvarez L, Dawson S, Cookson B, Hawkey P. Working across the veterinary and human health sectors. J Antimicrob Chemother (2012) 67:37-49. doi:10.1093/jac/dks206

6. Guardabassi L. Sixty years of antimicrobial use in animals: what is next? Vet Rec (2013) 173:599-603. doi:10.1136/vr.f7276

7. Van Boeckel TP, Brower C, Gilbert M, Grenfell BT, Levin SA, Robinson TP, et al. Global trends in antimicrobial use in food animals. Proc Natl Acad Sci US A (2015) 112(18):5649-54. doi:10.1073/pnas.1503141112

8. WHO Advisory Group on Integrated Surveillance of Antimicrobial Resistance (AGISAR). Critically Important Antimicrobials for Human Medicine - 3rd Revision 2011. World Health Organization (2011). p. 1-38. Available from: http://apps.who.int/iris/bitstream/10665/77376/1/9789241504485_eng.pdf

9. Nilsson O. Vancomycin resistant enterococci in farm animals - occurrence and importance. Infect Ecol Epidemiol (2012) 2:1-8. doi:10.3402/iee.v2i0.16959 
10. Geser N, Stephan R, Hächler H. Occurrence and characteristics of extended-spectrum $\beta$-lactamase (ESBL) producing Enterobacteriaceae in food producing animals, minced meat and raw milk. BMC Vet Res (2012) 8:21. doi:10.1186/1746-6148-8-21

11. Liu YY, Wang Y, Walsh TR, Yi LX, Zhang R, Spencer J, et al. Emergence of plasmid-mediated colistin resistance mechanism MCR-1 in animals and human beings in China: a microbiological and molecular biological study. Lancet Infect Dis (2016) 16:161-8. doi:10.1016/S1473-3099(15)00424-7

12. Chee-Sanford JC, Yannarell AC, Mackie RI. Environmental impacts of antibiotic use in the animal production industry. In: Leif N, Jeffrey L, editors. Ecosystem Health and Sustainable Agriculture, 2. Uppsala: Baltic University Programme (2012). p. 228-368.

13. Cabello FC, Godfrey HP, Buschmann AH, Dölz HJ. Aquaculture as yet another environmental gateway to the development and globalisation of antimicrobial resistance. Lancet Infect Dis (2016) 16:e127-33. doi:10.1016/ S1473-3099(16)00100-6

14. Chan M. Global Action Plan on Antimicrobial Resistance (Vol. 28). World Health Organization (2015). Available from: www.wpro.who.int/entity/ drug_resistance/resources/global_action_plan_eng.pdf

15. Lewis K. Platforms for antibiotic discovery. Nat Rev Drug Discov (2013) 12:371-87. doi:10.1038/nrd3975

16. Wood F. Antimicrobial Resistance and Medical Sociology: Research Brief. Bristol: University of Bristol (2016). Available at: http://www.bristol.ac.uk/ media-library/sites/social-community-medicine/documents/social-scienceand-amr/MedicalSociology\&AMR21092016.pdf

17. Carmo LP, Nielsen LR, Alban L, Müntener CR, Schüpbach G, Magouras I. Comparison of antimicrobial consumption patterns in the Swiss and Danish cattle and swine production (2007-2013). Front Vet Sci (2017) 4:1-11. doi: $10.3389 /$ fvets. 2017.00026

18. Grave K, Torren-Edo J, Mackay D. Comparison of the sales of veterinary antibacterial agents between 10 European countries. J Antimicrob Chemother (2010) 65:2037-40. doi:10.1093/jac/dkq247

19. Visschers VHM, Backhans A, Collineau L, Iten D, Loesken S, Postma M, et al. Perceptions of antimicrobial usage, antimicrobial resistance and policy measures to reduce antimicrobial usage in convenient samples of Belgian, French, German, Swedish and Swiss pig farmers. Prev Vet Med (2015) 119:10-20. doi:10.1016/j.prevetmed.2015.01.018

20. Cox G, Wright GD. Intrinsic antibiotic resistance: mechanisms, origins, challenges and solutions. Int J Med Microbiol (2013) 303:287-92. doi:10.1016/j. ijmm.2013.02.009

21. Singer AC, Shaw H, Rhodes V, Hart A. Review of antimicrobial resistance in the environment and its relevance to environmental regulators. Front Microbiol (2016) 7:1728. doi:10.3389/fmicb.2016.01728

22. EMA. Revised ESVAC Reflection Paper on Collecting Data on Consumption of Antimicrobial Agents per Animal Species, on Technical Units of Measurement and Indicators for Reporting Consumption of Antimicrobial Agents in Animals. London: European Medicines Agency (Vol. 44) (2013). p. 1-29. EMA/286416/2012-Rev1. Available from: http://www.ema.europa.eu/docs/ en_GB/document_library/Scientific_guideline/2012/12/WC500136456.pdf

23. EMA. ESVAC: vision, strategy and objectives 2016-2020. European Surveillance of Veterinary Antimicrobial Consumption. London: European Medicines Agency (2016). 44 p. Available from: http://www.ema.europa.
eu/docs/en_GB/document_library/Regulatory_and_procedural_guideline/2017/02/WC500221116.pdf

24. Bos MEH, Taverne FJ, van Geijlswijk IM, Mouton JW, Mevius DJ, Heederik DJJ. Consumption of antimicrobials in pigs, veal calves, and broilers in the Netherlands: quantitative results of nationwide collection of data in 2011. PLoS One (2013) 8:e77525. doi:10.1371/journal.pone.0077525

25. Stege H, Bager F, Jacobsen E, Thougaard A. VETSTAT - the Danish system for surveillance of the veterinary use of drugs for production animals. Prev Vet Med (2003) 57:105-15. doi:10.1016/S0167-5877(02)00233-7

26. Speksnijder DC, Mevius DJ, Bruschke CJM, Wagenaar JA. Reduction of veterinary antimicrobial use in the Netherlands. The Dutch success model. Zoonoses Public Health (2015) 62:79-87. doi:10.1111/zph.12167

27. Jensen VF, de Knegt LV, Andersen VD, Wingstrand A. Temporal relationship between decrease in antimicrobial prescription for Danish pigs and the "Yellow Card" legal intervention directed at reduction of antimicrobial use. Prev Vet Med (2014) 117:554-64. doi:10.1016/j.prevetmed. 2014.08.006

28. Carmo LP, Schüpbach-Regula G, Müntener C, Chevance A, Moulin G, Magouras I. Approaches for quantifying antimicrobial consumption per animal species based on national sales data: a Swiss example (2006-2013). Euro Surveill (2017) 22(6):30458. doi:10.2807/1560-7917.es.2017.22.6.30458

29. Crombé F, Argudín MA, Vanderhaeghen W, Hermans K, Haesebrouck F, Butaye P. Transmission dynamics of methicillin-resistant Staphylococcus aureus in pigs. Front Microbiol (2013) 4:57. doi:10.3389/fmicb.2013.00057

30. Espinosa-Gongora C, Broens EM, Moodley A, Nielsen JP, Guardabassi L. Transmission of MRSA CC398 strains between pig farms related by trade of animals. Vet Rec (2012) 170:564-564. doi:10.1136/vr.100704

31. Andraud M, Rose N, Laurentie M, Sanders P, Le Roux A, Cariolet R, et al. Estimation of transmission parameters of a fluoroquinolone-resistant Escherichia coli strain between pigs in experimental conditions. Vet Res (2011) 42:44. doi:10.1186/1297-9716-42-44

32. Heuer H, Schmitt H, Smalla K. Antibiotic resistance gene spread due to manure application on agricultural fields. Curr Opin Microbiol (2011) 14:236-43. doi:10.1016/j.mib.2011.04.009

33. Gordon DM. Geographical structure and host specificity in bacteria and the implications for tracing the source of coliform contamination. Microbiology (2001) 147:1079-85. doi:10.1099/00221287-147-5-1079

34. Carroll D, Wang J, Fanning S, McMahon BJ. Antimicrobial resistance in wildlife: implications for public health. Zoonoses Public Health (2015) 62:534-42. doi:10.1111/zph.12182

Conflict of Interest Statement: The authors declare that the research was conducted in the absence of any commercial or financial relationships that could be construed as a potential conflict of interest.

Copyright (C) 2017 Magouras, Carmo, Stärk and Schüpbach-Regula. This is an open-access article distributed under the terms of the Creative Commons Attribution License (CC BY). The use, distribution or reproduction in other forums is permitted, provided the original author(s) or licensor are credited and that the original publication in this journal is cited, in accordance with accepted academic practice. No use, distribution or reproduction is permitted which does not comply with these terms. 